\title{
Impactos da pandemia da COVID-19 no ensino teórico-prático da graduação em enfermagem
}

\author{
Impacts of the COVID-19 pandemic on theoretical-practical teaching of nursing graduation \\ Impactos de la pandemia COVID-19 en la enseñanza teórico-práctica de la graduación en \\ enfermería
}

Recebido: 15/03/2021 | Revisado: 21/03/2021 | Aceito: 27/03/2021 | Publicado: 04/04/2021

\author{
Suzana Pereira Alves \\ ORCID: https://orcid.org/0000-0003-1822-9762 \\ Christus Faculdade do Piauí, Brasil \\ E-mail: suzaninhaalves10@gmail.com \\ José Marcos Fernandes Mascarenhas \\ ORCID: https://orcid.org/0000-0002-2604-4330 \\ Christus Faculdade do Piauí, Brasil \\ E-mail: zemarcosmascarenhas@gmail.com \\ Luis Henrique Araújo Andrade \\ ORCID: https://orcid.org/0000-0002-3504-0710 \\ Christus Faculdade do Piauí, Brasil \\ E-mail: luishenriqueenf22@gmail.com \\ Iasmim Escórcio de Brito Melo \\ ORCID: https://orcid.org/0000-0002-5984-0309 \\ Christus Faculdade do Piauí, Brasil \\ E-mail: Iasmimedb@gmail.com \\ Antonia Mylene Sousa Almeida \\ ORCID: https://orcid.org/0000-0003-2695-6505 \\ Faculdade de Educação São Francisco, Brasil \\ E-mail: mylenesousa123@hotmail.com \\ Maria Rosemary da Silva Gomes \\ ORCID: https://orcid.org/0000-0002-8217-3647 \\ Centro Universitário Santo Agostinho, Brasil \\ E-mail: mariarosemary2010@gmail.com \\ Juliana da Silva Sousa \\ ORCID: https://orcid.org/0000-0002-5653-9473 \\ Christus Faculdade do Piauí, Brasi \\ E-mail: binhojuhkethy19@gmail.com \\ Allana Gabrielly da Silva Brasil Celestino \\ ORCID: https://orcid.org/0000-0002-5133-716X \\ Christus Faculdade do Piauí, Brasil \\ E-mail: allanag937@gmail.com \\ José Gabriel Fontenele Gomes \\ ORCID: https://orcid.org/0000-0001-6114-0726 \\ Farmacêutico, Brasil \\ E-mail: jgabrielfontenele@gmail.com \\ Anne Heracléia de Brito e Silva \\ ORCID: https://orcid.org/0000-0002-3414-8308 \\ Christus Faculdade do Piauí, Brasil \\ E-mail: Anneheracleiabs@hotmail.com
}

\begin{abstract}
Resumo
O presente estudo tem por objetivo reconhecer os impactos da pandemia na formação dos estudantes da graduação em enfermagem, em primeiro momento, a fim de questionar e evidenciar o cenário atual em que os estudantes se encontram; como a impossibilidade do contato físico prejudicou as práticas laboratoriais e de estágio, consequentemente, como isso afetou a formação dos mesmos, enquanto futuros profissionais de enfermagem. Para tanto realizou-se uma revisão integrativa da literatura, cuja relevância consiste na oportunidade para reunir, revisar, atualizar e discutir temas importantes e em voga. Diante do cenário pandêmico e a necessidade de isolamento social em detrimento do COVID-19, foram necessárias alterações concernentes às formas de ensino, tanto na graduação, quanto na pós-graduação. Nesse contexto, verificou-se limitações significativas, tanto no que confere ao processo de ensino-aprendizagem quanto ao cuidado com a doença em si. Ademais, é válido ressaltar que a modalidade exclusivamente à distância - apesar de os recursos em EAD - não torna possível suprir os elementos primordiais no
\end{abstract}


cuidado, como o contato direto, a interação face a face, a presença; ações que desde sempre, compõem a enfermagem. Dessa forma, o artigo contribui para a disseminação de informações acerca do tema em questão, além de contribuir para a melhoria das questões voltadas para a oferta de uma maior qualidade no desenvolvimento profissionalizante de novos profissionais de enfermagem.

Palavras-chave: COVID-19; Enfermagem; Ensino.

\begin{abstract}
The present study aims to recognize the impacts of the pandemic on the training of undergraduate nursing students, in the first instance, in order to question and highlight the current scenario in which the students find themselves; how the impossibility of physical contact hindered laboratory and internship practices, consequently, how it affected their training, as future nursing professionals. To this end, an integrative literature review was carried out, the relevance of which is the opportunity to gather, review, update and discuss important and fashionable topics. In view of the pandemic scenario and the need for social isolation to the detriment of COVID-19, changes were necessary concerning the forms of teaching, both in undergraduate and graduate courses. In this context, there were significant limitations, both in terms of the teaching-learning process and in caring for the disease itself. In addition, it is worth noting that the modality exclusively at a distance - despite the resources in distance education - does not make it possible to supply the essential elements in care, such as direct contact, face-to-face interaction, presence; actions that have always been part of nursing. Thus, the article contributes to the dissemination of information about the topic in question, in addition to contributing to the improvement of issues aimed at offering greater quality in the professional development of new nursing professionals.
\end{abstract}

Keywords: COVID-19; Nursing; Teaching.

\title{
Resumen
}

El presente estudio tiene como objetivo reconocer los impactos de la pandemia en la formación de estudiantes de licenciatura en enfermería, en primera instancia, con el fin de cuestionar y resaltar el escenario actual en el que se encuentran los estudiantes; cómo la imposibilidad de contacto físico entorpeció las prácticas de laboratorio y pasantías, en consecuencia, cómo afectó su formación como futuros profesionales de enfermería. Para ello, se realizó una revisión integradora de la literatura, cuya relevancia es la oportunidad de recopilar, revisar, actualizar y discutir temas importantes y de moda. Ante el escenario pandémico y la necesidad de aislamiento social en detrimento del COVID-19, fueron necesarios cambios en las formas de enseñanza, tanto en los cursos de pregrado como de posgrado. En este contexto, existían limitaciones importantes, tanto en el proceso de enseñanza-aprendizaje como en el cuidado de la propia enfermedad. Además, cabe señalar que la modalidad exclusivamente a distancia - a pesar de los recursos en educación a distancia - no permite suministrar los elementos esenciales en el cuidado, como el contacto directo, la interacción cara a cara, la presencia; acciones que siempre han formado parte de la enfermería. Así, el artículo contribuye a la difusión de información sobre el tema en cuestión, además de contribuir a la mejora de temas encaminados a ofrecer una mayor calidad en el desarrollo profesional de los nuevos profesionales de enfermería.

Palabras clave: COVID-19; Enfermería; Ensenãnza.

\section{Introdução}

A pandemia do COVID-19 decorrente do SARS-CoV-2 ou novo coronavírus, consiste em uma doença infecciosa, descoberta pela primeira vez na China, a partir de dezembro de 2019. Logo, devido à sua forte capacidade de transmissão, foi rapidamente reconhecido como uma emergência internacional de saúde pública. (Silva et al., 2021).

Atualmente, a maior medida para prevenir a propagação do vírus consiste no isolamento social. As autoridades fecharam locais como ambientes de lazer, propícios à convivência, permitindo a abertura apenas de locais estritamente necessários como supermercados, farmácias, e afins, seguiram as orientações da Organização Mundial de Saúde. Essas medidas afetaram a educação, tendo que se moldar para continuar suas atividades, seguindo os decretos impostos. (Pito \& Nunes, 2020).

Visto isso, após atingir o Brasil em março de 2020, fizeram-se necessárias respostas imediatas quanto à identificação dos mecanismos de transmissão do vírus. Diante desse contexto, foi denotada a necessidade do fornecimento de aportes para os alunos das áreas de saúde, principalmente os que se encontravam em situação de estágios avançados no processo de formação, para a manutenção da segurança na assistência e a qualidade de ensino (Prata et al., 2020). 
Outrossim, existem pressões causadas por diversos fatores, como o excesso de informações, que por vezes não são totalmente absolvidas pelos alunos; o risco de crise severa, bem como, a falta de garantia de manutenção da renda. Normalmente, os alunos também são profissionais em atividades práticas, enfrentam riscos profissionais, além de não sentirem confiança suficiente quanto ao seu rendimento ao realizarem atividades de forma remota, diante disso, acabam por não conseguirem produzir conhecimento, devido a mudança na rotina, distância social e famílias de risco. Todos esses fatores afetam diretamente o desempenho dos alunos (Silva et al., 2021).

Nesse sentido, o cenário atual de pandemia em que se encontra, prejudica diretamente o ensino dos estudantes. Além de outros fatores, para que seja posto em prática, essa modalidade pede que os alunos disponham de recursos, para que tenham acesso às aulas, recursos esses, que muitas vezes independem dos mesmos. Esse fato se explica, ao passo em que situações corriqueiras, como quedas de energia, oscilações na internet, nessa nova modalidade, podem comprometer o rendimento do dia (Cunha et al., 2020).

As dificuldades também são encontradas por parte dos professores, que encontram desafios para repassar o conteúdo de forma remota e principalmente, estão ligadas às atividades práticas e estágios. A premissa da formação do enfermeiro é estabelecer uma relação de confiança, a fim de proporcionar enfermagem e orientação na prática, tais aspectos constituem paradigmas que podem afetar a formação em enfermagem (Lira et al., 2020).

Pensando nas abordagens feitas, surgiu o questionamento: Quais os impactos da pandemia do COVID-19 no ensino teórico-prático da graduação em enfermagem? A pergunta norteadora impulsionou a construção desse estudo, tendo como objetivo, reconhecer os impactos da pandemia na formação dos estudantes da graduação em enfermagem, em primeiro momento, a fim de questionar e evidenciar o cenário atual em que os estudantes se encontram; como a impossibilidade do contato físico prejudicou as práticas laboratoriais e de estágio, consequentemente, como isso afetou a formação dos mesmos, enquanto futuros profissionais de enfermagem.

\section{Metodologia}

O artigo tem como metodologia utilizada a revisão integrativa da literatura, de abordagem qualitativa, cujo período de construção transcorreu agosto de 2020 a fevereiro de 2021 e envolveu a seleção e inclusão de artigos já publicados sobre a temática proposta. A relevância desse método é permitir a reunião, revisão, atualização e discussão de temas importantes e em voga na área da saúde. Ademais, fornece aos profissionais de saúde dados importantes de um específico assunto, em lugares e momentos diversos.

Segundo Souza, Silva e Carvalho (2010) a revisão integrativa propicia e determina a reunião atualizada do conhecimento sobre a temática a qual a revisão é dirigida. Envolve um rigor metodológico consistente que segue ao cumprimento de seis importantes etapas, sendo elas: preparar a pergunta norteadora, procurar ou pesquisar na literatura, reunir os dados, analisar de forma crítica os estudos incluídos, discutir os resultados, e por fim a apresentar a revisão integrativa.

A formulação da pergunta norteadora seguiu o uso da estratégia PICo, um método voltado a pesquisa não clínica, reconhecida como estratégia primordial em estudos de revisão integrativa. A distribuição dos caracteres segue a conformação: P - população, paciente (idade, sexo, status de saúde), problema; I - interesse; Co - contexto (Santos, Pimenta \& Nobre, 2007). Para o presente estudo considerou-se, P - impactos da pandemia do COVID-19, I - ensino teórico-prático e Co: graduação em enfermagem. Dessa forma, a pergunta de pesquisa ficou da seguinte forma: Quais os impactos da pandemia do COVID-19 no ensino teórico-prático da graduação em enfermagem?

O levantamento de artigos foi feito na biblioteca Scientific Electronic Library Online (SCIELO) e nas bases de dados da Literatura Latino-americana e do Caribe em Ciências da Saúde (LILACS), Sistema Online de Busca e Análise de Literatura 
Médica (MEDLINE) e a Base de Dados de Enfermagem (BDENF) via Biblioteca Virtual em Saúde (BVS). Para a operacionalização da busca foram casados com o operador booleano AND os seguintes descritores em português: COVID-19 AND Enfermagem AND Educação; e em inglês: COVID-19 AND Nursing AND Education.

Somente foram incluídos ao estudo os artigos completos de origem nacional e internacional, disponíveis gratuitamente na íntegra nos idiomas português e inglês, que contemplassem a temática e o objetivo proposto, assim como solução à pergunta norteadora. Por conta de a temática ser nova, não houve necessidade de aplicar o critério de recorte temporal, uma que vez que os estudos voltados a temática em questão mostram-se escassos. Ademais, excluísse os artigos incompletos, duplicados, pagos, com distanciamento da proposta temática e do objetivo proposto. Ressalta-se que foram respeitados todos os aspetos éticos legais, uma vez que somente foram utilizados dados de domínio público, sendo dada a devida referência as fontes autorais.

Ao realizar a busca, inicialmente foram revelados 14 artigos científicos na Biblioteca Scielo; 43 artigos na base BDENF; 44 artigos na base LILACS e 127 artigos na base MEDLINE. A coleta de dados foi efetuada primeiramente com uma leitura exploratória e rápida de todo material de estudo selecionado, em seguida, uma leitura seletiva para a escolha dos artigos de maior interesse, na qual estes foram lidos integralmente, totalizando 18 artigos, e por fim, uma leitura analítica destes artigos, na qual 13 foram escolhidos para fazer parte deste estudo, no tocante que atendiam à questão norteadora. As etapas desta busca estão descritas na Tabela 1.

Tabela 1 - Distribuição dos artigos obtidos na biblioteca Scielo e nas bases de dados BDENF, LILACS e MEDLINE, segundo os descritores selecionados.

\begin{tabular}{c|c|c|c|c}
\hline BASE DE DADOS & DESCRITORES & $\begin{array}{c}\text { ARTIGOS } \\
\text { OBTIDOS }\end{array}$ & $\begin{array}{c}\text { ARTIGOS } \\
\text { ANALISADOS }\end{array}$ & $\begin{array}{c}\text { ARTIGOS SELECIONADOS } \\
\text { PARA O ESTUDO }\end{array}$ \\
\hline SCIELO & $\begin{array}{c}\text { COVID-19 AND } \\
\text { enfermagem AND } \\
\text { graduação }\end{array}$ & 14 & 3 & 3 \\
\hline BDENF & $\begin{array}{c}\text { COVID-19 AND } \\
\text { enfermagem AND } \\
\text { educação }\end{array}$ & 43 & 2 & 8 \\
\hline LILACS & $\begin{array}{c}\text { COVID-19 AND } \\
\text { enfermagem AND } \\
\text { educação }\end{array}$ & 44 & 12 & 1 \\
\hline MEDLINE & $\begin{array}{c}\text { COVID-19 AND } \\
\text { enfermagem AND } \\
\text { educação }\end{array}$ & 127 & 1 & 1 \\
\hline
\end{tabular}

Fonte: Autores (2021).

Os artigos selecionados foram posteriormente submetidos à análise temática (AT). Este método de análise qualitativa de dados tem como objetivo a identificação, análise, interpretação e relatos de padrões (temas) através de dados qualitativos. Ademais, é possível descrever e organizar as informações detalhadamente, além de analisá-los de forma interpretativa.

A AT pode ser utilizada de duas formas: por meio de uma abordagem dedutiva ou teórica- temas bem definidos com um conjunto de categorias preestabelecidas; ou por abordagem indutiva e baseada nos dados- não parte de temas ou categorias prontas para analisar dados. Todavia, em ambas abordagens, a AT oferece ampla praticidade e aplicabilidade, no tocante que pode ser utilizada em quase todos estudos de análise qualitativa (Souza, 2019). 


\section{Resultados e Discussão}

Para uma melhor organização dos resultados e da análise dos dados, no Quadro 1 estão dispostas as principais informações dos 13 artigos incluídos neste artigo de revisão integrativa. Esta disposição de dados considerou apresentar as seguintes informações: autoria e ano de publicação, objetivo, tipo de estudo e conclusão do artigo.

Quadro 1 - Distribuição dos artigos inclusos no presente trabalho.

\begin{tabular}{|c|c|c|c|}
\hline Autoria e ano de publicação & Objetivo & Tipo de estudo & Conclusão do artigo \\
\hline (Prata et al, 2020) & $\begin{array}{l}\text { Relatar a experiência do } \\
\text { desenvolvimento de mediações } \\
\text { pedagógicas em Ambiente } \\
\text { Virtual de Aprendizagem } \\
\text { implementadas em uma } \\
\text { faculdade de enfermagem } \\
\text { durante a pandemia de COVID- } \\
19 .\end{array}$ & $\begin{array}{ll}\text { Relato } & \text { de } \\
\text { experiência }\end{array}$ & $\begin{array}{l}\text { Experimentar a educação não formal foi uma } \\
\text { experiência que forneceu saberes essenciais } \\
\text { para o planejamento do ensino formal em um } \\
\text { futuro de retorno à vida universitária, esses } \\
\text { desafios esbarram na presencialidade, } \\
\text { considerando as particularidades } \\
\text { epidemiológicas da COVID-19 e as deficiências } \\
\text { estruturais das universidades no Brasil. }\end{array}$ \\
\hline (Peres et al., 2020) & $\begin{array}{l}\text { Apresentar argumentos } \\
\text { reflexivos sobre as medidas de } \\
\text { aceleração da formação de } \\
\text { enfermeiros e inserção precoce } \\
\text { no sistema de saúde durante a } \\
\text { pandemia da COVID-19. }\end{array}$ & $\begin{array}{l}\text { Texto analítico, } \\
\text { teórico-reflexivo }\end{array}$ & $\begin{array}{l}\text { A inclusão de estudantes do curso de graduação } \\
\text { na atuação em áreas prioritárias, no } \\
\text { enfrentamento da epidemia, certamente traria } \\
\text { benefícios para as equipes uma vez que renova a } \\
\text { força de trabalho, ademais inclui profissionais } \\
\text { mais jovens que, em sua maioria, não estariam } \\
\text { no grupo de risco para as formas graves da } \\
\text { doença. Entretanto, os riscos são altos sejam em } \\
\text { relação a decisão clínica, ao contrapasso de } \\
\text { dilemas e processos éticos. }\end{array}$ \\
\hline (Cunha et al., 2020) & $\begin{array}{l}\text { Analisar as ações de ensino, } \\
\text { pesquisa, extensão, assistência e } \\
\text { gestão para o enfrentamento da } \\
\text { pandemia da COVID-19, no } \\
\text { âmbito de Escolas e } \\
\text { Departamentos de Enfermagem } \\
\text { de Universidades Federais } \\
\text { brasileiras. }\end{array}$ & $\begin{array}{l}\text { Pesquisa } \\
\text { documental }\end{array}$ & $\begin{array}{l}\text { Escolas e departamentos de enfermagem } \\
\text { desenvolveram ações com alinhamento as } \\
\text { universidades, mostraram ação e } \\
\text { responsabilidade social com a comunidade, } \\
\text { tanto nas atividades de gestão, quanto de ensino, } \\
\text { pesquisa e extensão, cuja organização foi de } \\
\text { forma rápida e eficaz, por meio da } \\
\text { reorganização dos processos de trabalho. }\end{array}$ \\
\hline (Geremia et al., 2020) & $\begin{array}{l}\text { Compreender a atuação do } \\
\text { enfermeiro no Sistema Unico de } \\
\text { Saude frente ao Coronavirus } \\
\text { Disease } 2019 \text { e sua relação com } \\
\text { o processo de formação } \\
\text { profissional. }\end{array}$ & Estudo qualitativo & $\begin{array}{l}\text { O fenômeno da COVID-19 fez com que se } \\
\text { emergisse pontos de vista técnico e científico no } \\
\text { processo de formar e produzir conhecimento } \\
\text { científico, que requer revisar o papel primordial } \\
\text { do Estado em garantir o direito público e } \\
\text { universal da saúde por meio do SUS, as formas } \\
\text { de trabalho dos profissionais, a integração de } \\
\text { instrução e serviço para a formar enfermeiros } \\
\text { que problematizam e ocupam papéis de } \\
\text { liderança, com presteza para atuar diante das } \\
\text { funções gestoras, assistenciais, pesquisadora, } \\
\text { educadora e política. }\end{array}$ \\
\hline (Agu et al., 2020) & $\begin{array}{l}\text { Descrever o efeito do COVID - } \\
19 \text { na educação de enfermagem } \\
\text { em países em desenvolvimento. }\end{array}$ & Estudo reflexivo & $\begin{array}{l}\text { O impacto da pandemia COVID - } 19 \text { na } \\
\text { educação de enfermagem em países em } \\
\text { desenvolvimento pode ser maior do que em } \\
\text { países desenvolvidos devido às disparidades. O } \\
\text { aprendizado online se tornou a solução para } \\
\text { completar o currículo; no entanto, não aborda o } \\
\text { componente de prática clínica. }\end{array}$ \\
\hline
\end{tabular}




\begin{tabular}{|c|c|c|c|}
\hline (Lira et al., 2020) & $\begin{array}{l}\text { Discutir sobre os desafios e } \\
\text { perspectivas da educação em } \\
\text { enfermagem em tempos da } \\
\text { pandemia COVID-19. }\end{array}$ & Estudo reflexivo & $\begin{array}{l}\text { Desafios de longa data da educação em } \\
\text { enfermagem ficaram mais evidentes com a } \\
\text { pandemia, dessa mesma forma foram os } \\
\text { métodos de aceleração, alteração e interrupção } \\
\text { na educação. Para o ensino de enfermagem a } \\
\text { necessidade emergencial de tecnologias de } \\
\text { interface que ajustem o físico com o digital, } \\
\text { ampliem a discussão, as trocas de experiências, } \\
\text { interações, reflexões e o pensamento crítico. }\end{array}$ \\
\hline (Motta et al., 2021) & $\begin{array}{l}\text { Descrever o desenvolvimento } \\
\text { de um objeto de aprendizagem } \\
\text { focado em evidências } \\
\text { científicas sobre COVID-19. }\end{array}$ & $\begin{array}{l}\text { Relato de } \\
\text { experiência }\end{array}$ & $\begin{array}{l}\text { O objeto de aprendizagem é um site, cuja } \\
\text { proposta responde a metas estabelecidas pela } \\
\text { campanha Nursing Now, que trata do } \\
\text { investimento na educação disseminando } \\
\text { evidências científicas passíveis de aplicar na } \\
\text { prática. Ademais, o design intuitivo do site, } \\
\text { possui uma linguagem prática e de fácil } \\
\text { entendimento, que aceita consultas rápidas, com } \\
\text { acesso ao que trata das melhores recomendações } \\
\text { sobre prevenção, promoção e conservação da } \\
\text { saúde, além de explicações sobre COVID-19 } \\
\text { aos profissionais de saúde e população em geral. }\end{array}$ \\
\hline (Bastos et al., 2020) & $\begin{array}{l}\text { Descrever a experiência no } \\
\text { ensino remoto emergencial para } \\
\text { as aulas teóricas na graduação } \\
\text { em } \quad \text { Enfermagem } \quad \text { em } \\
\text { decorrência da COVID-19 }\end{array}$ & $\begin{array}{l}\text { Relato } \\
\text { experiência }\end{array}$ & $\begin{array}{l}\text { O ensino remoto ultrapassou a aptidão e } \\
\text { assistência dos professores e alunos quanto o } \\
\text { emprego de recursos virtuais, bem como pela } \\
\text { urgência de readequação dos métodos de } \\
\text { ensino, as quais alternaram desde a execução de } \\
\text { conferências virtuais até a produção agrupada } \\
\text { de artefatos que constituíram atividades } \\
\text { avaliativas nesse local. }\end{array}$ \\
\hline $\begin{array}{c}\text { (Soccol, Santos e Marchiori, } \\
\text { 2020) }\end{array}$ & $\begin{array}{lrr}\text { Refletir } & \text { sobre as } & \text { contribuições } \\
\text { do } & \text { estágio } & \text { curricular } \\
\text { supervisionado } & \text { no } & \text { contexto da } \\
\text { COVID-19 } & \text { para } & \text { o } \\
\text { desenvolvimento } & \text { profissional } \\
\text { dos estudantes de } & \text { Enfermagem. }\end{array}$ & Estudo reflexivo & $\begin{array}{l}\text { O estágio supervisionado no cenário da } \\
\text { pandemia referente as tarefas da Atenção } \\
\text { Básica, fortifica a educação e o progresso } \\
\text { profissional dos acadêmicos e concede } \\
\text { visibilidade à importância da profissão. }\end{array}$ \\
\hline (Bezerra, 2020) & $\begin{array}{l}\text { Descrever o estado da arte sobre } \\
\text { o ensino de enfermagem e os } \\
\text { desafios do uso de tecnologias } \\
\text { remotas em época de pandemia } \\
\text { do Corona vírus. }\end{array}$ & Estudo reflexivo & $\begin{array}{l}\text { Com a exigência da inserção desses recursos } \\
\text { para a prosseguimento de aulas no modelo não } \\
\text { presencial pôde possibilitar a se ter uma nova } \\
\text { visibilidade sobre o assunto, com oportunidade } \\
\text { de expandir o debate sobre uso dessas } \\
\text { ferramentas remotas na docência em saúde, } \\
\text { almejando uma reflexão sobre a relação destas } \\
\text { com os outros métodos de educação já } \\
\text { realizados. }\end{array}$ \\
\hline (Souza et al., 2020) & $\begin{array}{l}\text { Relatar as experiências de } \\
\text { estudantes de enfermagem } \\
\text { durante o estágio curricular } \\
\text { supervisionado na atenção } \\
\text { básica no cenário da pandemia } \\
\text { de Coronavírus. }\end{array}$ & $\begin{array}{l}\text { Relato } \\
\text { experiência }\end{array}$ & $\begin{array}{l}\text { Os estágios supervisionados realizados em } \\
\text { condições de pandemia capacitam os alunos, } \\
\text { proporcionam novas aprendizagens e } \\
\text { experiencias impostas no momento, são } \\
\text { propícios ao crescimento pessoal, em termos de } \\
\text { tomada de decisão, autonomia e liderança. }\end{array}$ \\
\hline $\begin{array}{l}\text { (Scorsolini-Comin et al., } \\
\text { 2020) }\end{array}$ & $\begin{array}{l}\text { Refletir sobre o emprego da } \\
\text { educação a distância } \\
\text { graduação em enfermagem no } \\
\text { Brasil no cenário da pandemia } \\
\text { da COVID-19. }\end{array}$ & Ensaio crítico & $\begin{array}{l}\text { As metodologias de ensino a distância permitem } \\
\text { a continuação do processo acadêmico de } \\
\text { enfermagem. Reitera-se que o ensino e } \\
\text { aprendizagem do cuidado em saúde requer } \\
\text { contato e proximidade. }\end{array}$ \\
\hline
\end{tabular}




\begin{tabular}{|l|l|l|l|}
\hline (Silva et al., 2021) & $\begin{array}{l}\text { Discutir as atividades não } \\
\text { presenciais no ensino de } \\
\text { enfermagem, no contexto da } \\
\text { pandemia da COVID-19 e em } \\
\text { meio à campanha "Nursing } \\
\text { Now" pelo fortalecimento da } \\
\text { enfermagem. }\end{array}$ & & \\
crítica & & $\begin{array}{l}\text { Evidencia-se que os enfermeiros possuem } \\
\text { perdas no ensino emergencial realizado a } \\
\text { distância, em contrapartida ao movimento } \\
\text { internacional pela valorização da enfermagem. } \\
\text { Nesse sentido, é importante usufruir das } \\
\text { tecnologias virtuais ligadas ao ensino, mesmo } \\
\text { que esta metodologia de ensino remoto não seja } \\
\text { suficiente para o ensino em enfermagem de } \\
\text { forma integral. }\end{array}$ \\
\hline
\end{tabular}

Fonte: Autores (2021).

Diante do cenário pandêmico e a necessidade de isolamento social em detrimento do COVID-19, foram necessárias alterações concernentes às formas de ensino, tanto na graduação, quanto na pós-graduação. Dessa forma, foi realizado também, em alguns âmbitos, o cancelamento das aulas presenciais. Visto isso, aumentou-se sobre professores e gestores a pressão por garantir a qualidade na formação e atender os requisitos exigidos por esta.

Como estratégia de continuidade no desenvolvimento da aprendizagem no ensino superior, foi então implementada a modalidade remota, com a utilização de plataformas que permitem a interação em tempo real. Nesse modelo, os professores passaram a utilizar fóruns de pergunta e espaços para sanar as dúvidas dos alunos. Dessa maneira, os educadores mantêm o processo avaliativo, respeitando o isolamento social e o adiamento das atividades presenciais até que se obtenha o controle da pandemia.

Apesar da rápida construção de ações para o salteamento do novo coronavírus implementada pelas universidades durante o início da pandemia, verificou-se limitações significativas, tanto no que confere ao processo de ensino-aprendizagem quanto ao cuidado com a doença em si. Ademais, isso pode ser relacionado à pouca disseminação de conhecimentos acurados e domínio específico da doença, bem como, a prática de cuidados inerentes à esta.

Nesse sentido, apesar de o ensino remoto ter sido adotado atualmente como uma saída urgente, a fim de que se possam continuar os processos de ensino aprendizagem e até mesmo, para contribuir para que estudantes pudessem se formar e atuarem também nas linhas de frente, é válido ressaltar que a modalidade exclusivamente à distância - apesar dos recursos em ensino à distância (EAD) - não torna possível suprir os elementos primordiais no cuidado, como o contato direto, a interação face a face, a presença; ações que desde sempre, compõem a enfermagem.

Outrossim, é sabido que dentro da enfermagem, as relações de troca entre paciente e profissional são primordiais para a formação de futuros profissionais aptos a executar um atendimento humanizado. Dessa forma, destaca-se outro desafio dentro do cenário EAD, uma vez que, os recursos tecnológicos para aulas não presenciais, tendem a afastar os alunos das atividades práticas, retirando a prática das atividades com o convívio, trazendo possíveis consequenciais nos atendimentos desses futuros profissionais.

Dessa forma, com base na revisão dos artigos publicados, é possível afirmar que os métodos de ensino remoto apresentam pontos positivos no que se refere à preparação dos alunos e docentes quanto ao manejo das ferramentas digitais. No entanto, com a mudança brusca no cenário de ensino, a readequação e a revisão das metodologias de ensino se deram de maneira forçada, dessa forma, refletindo sobre a qualidade do processo de formação acadêmica.

Por outro lado, denotou-se a importância do estágio supervisionado durante o ensino emergencial, pois além de favorecer o raciocínio crítico e o amadurecimento dos discentes no quesito profissional, também há a preservação de profissionais que se encontram em situação de risco. Porém, a equipe perde em relação ao poder das tomadas de decisão, que por sua vez, são cruciais para um bom prognóstico dos pacientes. 


\section{Conclusão}

É notória a dificuldade enfrentada tanto por parte dos docentes, como principalmente dos acadêmicos de Enfermagem perante o cenário pandêmico, haja vista que é exigido um grande esforço para a adaptação destes às novas formas de ensino contemporâneas. Por conseguinte, ter que lidar com contratempos, muitas das vezes, perpassam a capacidade de resolução de problemas convencionais. Por outro lado, a submissão de condições adversas permite que o binômio do proc esso educacional seja aperfeiçoado e moldado para as versões de um novo ponto de vista.

Portanto, conclui-se que o artigo aborda de forma sucinta os impactos da pandemia da COVID-19 no ensino teóricoprático da graduação em enfermagem, bem como a readequação de novas metodologias de ensino utilizadas na modalidade remota e seus impactos no processo de ensino-aprendizagem durante a formação destes. Dessa forma, contribuindo para a disseminação de informações acerca do tema em questão, além de contribuir para a melhoria das questões voltadas para a oferta de uma maior qualidade no desenvolvimento profissionalizante de novos profissionais de enfermagem.

Reitera-se ainda, que mediante o exposto, fica claro a relevância do tema desse estudo e salienta-se a importância de mais estudos serem feitos acerca da questão, onde estes devem buscar ressaltar a relação entre as metodologias remotas utilizadas e como estas tendem a ser gatilhos para um leque de desafios que surgem para a comunidade como um todo e principalmente, acadêmica e profissional.

\section{Referências}

Agu, C. F. et al. (2021). COVID-19 pandemic effects on nursing education: looking through the lens of a developing country. International Nursing Review, 1(1), 1-6. https://onlinelibrary.wiley.com/doi/epdf/10.1111/inr.12663

Bastos, M. C., et al. (2020). Ensino remoto emergencial na graduação em enfermagem: relato de experiência na covid-19. Revista Mineira de Enfermagem, 24(1), 1-6. https://cdn.publisher.gn1.link/reme.org.br/pdf/e1335.pdf

Bezerra, I. M. P. (2020). Estado da arte sobre o ensino de enfermagem e os desafios do uso de tecnologias remotas em época de pandemia do coronavírus. Revista Brasileira do Crescimento e Desenvolvimento Humano, 30(1), 141-147. http://doi.org/10.7322/jhgd.v30.10087

Cunha, I. C. K. O., et al. (2020). Ações e estratégias de escolas e departamentos de enfermagem de universidades federais frente à COVID-19. Enfermagem em foco, 11(1), 48-57. http://revista.cofen.gov.br/index.php/enfermagem/article/view/4115/802

Geremia, D. S., et al. (2020). Pandemia covid-2019: formação e atuação da enfermagem para o Sistema Único de Saúde. Enfermagem em foco, 11(1), 40-47. http://revista.cofen.gov.br/index.php/enfermagem/article/view/4115/802

Lira, A. L. B. C., et al. (2020). Educação em enfermagem: desafios e perspectivas em tempos da pandemia. Revista Brasileira de Enfermagem, 73(2), 1-6. https://doi.org/10.1590/0034-7167-2020-0683

Motta, L. D., et al. (2021). COVID-19 evidências para todos: desenvolvimento de um objeto de aprendizagem no ensino em saúde. Revista Gaúcha de Enfermagem, 42(1), 1-10. https://doi.org/10.1590/1983-1447.2021.20200281

Peres, M. A. A., et al. (2020). Enfrentamento da covid-19: o que não pode ser relativizado na educação superior em enfermagem. Texto contexto enfermagem, 29(1), 1-13. https://doi.org/10.1590/1980-265x-tce-2020-0236

Prata, J. A., et al. (2020). Mediações pedagógicas para o ensino não formal de enfermagem durante a pandemia COVID-19. Revista Brasileira de Enfermagem, 73(1), 1-5. http://dx.doi.org/10.1590/0034-7167-2020-0499

Pito, L. B. S., \& Nunes, M. I. (2020). Problematização sobre a pandemia da COVID-19 como auxílio na formação de enfermeiras/os. Revista Nursing, 23(266), 4294-4300. http://www.revistas.mpmcomunicacao.com.br/index.php/revistanursing/login?source=\%2Findex.php\%2Frevistanursing\%2Farticle $\% 2$ Fview\%2F789

Santos, C. M. C., Pimenta, C. A. M., \& Nobre, M. R. C. A. (2007). A estratégia pico para a construção da pergunta de pesquisa e busca de evidências. Revista Latino-Americana de Enfermagem, 15(3), 1-3. https://www.scielo.br/pdf/rlae/v15n3/pt_v15n3a23.pdf

Scorsolini-Comin, F., et al. (2020). Educação a distância na formação em enfermagem: reflexões sobre a pandemia da covid-19. Revista Baiana de Enfermagem, 34(1), 1-9. http://dx.doi.org/10.18471/rbe.v34.36929

Silva, C. M., et al. (2021). Pandemia da COVID-19, ensino emergencial a distância e Nursing Now: desafios à formação em enfermagem. Revista Gaúcha de Enfermagem, 42(1), 1-13. https://doi.org/10.1590/1983-1447.2021.20200248

Soccol, K. L. S., Santos, N. O., \& Marchiori, M. R. C. T. Estágio Curricular Supervisionado no contexto da COVID-19 e o desenvolvimento profissional de estudantes de Enfermagem. Enfermagem em Foco, 11(2), 148-151. http://revista.cofen.gov.br/index.php/enfermagem/article/view/4173/998 
Research, Society and Development, v. 10, n. 4, e18210413924, 2021

(CC BY 4.0) | ISSN 2525-3409 | DOI: http://dx.doi.org/10.33448/rsd-v10i4.13924

Souza, L. B., et al. (2020). Estágio curricular supervisionado em enfermagem durante a pandemia de Coronavírus: experiências na atenção básica. J. nurs. Health, 10(1), 1-10. https://doi.org/10.15210/jonah.v10i4.19050

Souza, L. K. Pesquisa com análise qualitativa de dados: conhecendo a Análise Temática. Arquivos Brasileiros de Psicologia, 71(2), 51-67. http://pepsic.bvsalud.org/pdf/arbp/v71n2/05.pdf

Souza, M. T., Silva, M. D., \& Carvalho, R. Revisão integrativa: o que é e como fazer. Einstein, 8(1), 102-106. https://www.scielo.br/pdf/eins/v8n1/pt_16794508-eins-8-1-0102.pdf 